\title{
Bronchoalveolar lavage cellularity in infants with severe respiratory syncytial virus bronchiolitis
}

\author{
P S McNamara, P Ritson, A Selby, C A Hart, R L Smyth
}

Arch Dis Child 2003;88:922-926

\begin{abstract}
Aim: To examine over time, the cellular response within the lungs of infants ventilated with respiratory syncytial virus (RSV) bronchiolitis and to compare this response in infants born at term with those born preterm.

Methods: Non-bronchoscopic bronchoalveolar lavage (BAL) samples were taken from 47 infants (24

born at term and 23 born preterm) who were ventilated for RSV positive bronchiolitis and 10 control infants. BAL cellularity and differential cell counts were calculated using standard techniques.

Results: Total cellularity in BAL over the first four days of ventilation in infants with RSV bronchiolitis was greater in term infants (median 2.2 (IQR 4.27) $\times 10^{6}$ cells $/ \mathrm{ml}$ ) compared with preterm infants $\left(0.58(1.28) \times 10^{6} \mathrm{cell} / \mathrm{s} / \mathrm{ml}\right)$. The magnitude of the cellular response in preterm infants with bronchiolitis was similar to that in the control group measured on day $1\left(0.62(0.77) \times 10^{6}\right.$ cells $\left./ \mathrm{ml}\right)$. BAL cellularity decreased progressively from the time of intubation in term infants, but remained relatively constant in preterm infants up to seven days after intubation.

Conclusions: There are differences in the magnitude and type of pulmonary cellular response in term and preterm infants ventilated with RSV bronchiolitis. The cellular response in term infants with bronchiolitis differs from that in a control group of infants. These differences may reflect variations in cellular recruitment in the lung and/or variations in airway calibre.
\end{abstract}

See end of article for authors' affiliations

Correspondence to:

Professor R Smyth,

Department of Child

Health, Alder Hey

Children's Hospital, Eaton

Rd, Liverpool L12 2AP, UK;

r.l.smyth@liv.ac.uk

Accepted

17 January 2003
$\mathrm{R}$ espiratory syncytial virus (RSV) bronchiolitis is one of the most important causes of death and morbidity in infants throughout the world. In urban populations $0.8-2 \%$ of infants have symptoms severe enough to warrant hospitalisation, ${ }^{1-3}$ and of these around $2-3 \%$ develop respiratory failure and require ventilation. ${ }^{13}$ Treatment for RSV bronchiolitis is largely supportive and there is currently no effective vaccine. ${ }^{14}$ Factors that predispose to severe disease include prematurity. ${ }^{1}$ Although preterm infants account for only $6 \%$ of all births, they represent up to $24 \%$ of infants hospitalised for bronchiolitis and up to $63 \%$ of infants ventilated for bronchiolitis. ${ }^{5}$ Preterm infants may be at particular risk of severe RSV disease for a number of reasons. It has been suggested that because of their immature immune responses and lack of maternally derived RSV neutralising antibody, preterm infants are more likely to acquire to RSV infection and less likely to eradicate the virus once infected. ${ }^{7}$ They may also have underlying pathological changes caused by arrested pulmonary development (the so called "new BPD") or secondary to barotrauma and oxidant injury. ${ }^{89}$

While the cellular and immunological responses to RSV infection in the lung have been well documented in animal models, ${ }^{10-13}$ there are few studies that have examined these responses in infants with RSV bronchiolitis. Two studies have shown that neutrophils are the predominant cellular infiltrate in the airways of infants with RSV bronchiolitis. ${ }^{14}{ }^{15}$ In contrast, specimens of lung taken from infants with respiratory viral infections at postmortem examination describe a lymphocytic peribronchiolar infiltrate as being characteristic of histological bronchiolitis. ${ }^{16}$ In those infants who died as a result of the RSV vaccine augmented disease in the 1960s, an eosinophilic infiltrate was described in the lungs. ${ }^{17}$

We wished to study the cellular response in the lungs of infants with severe RSV bronchiolitis and to investigate how this changed over time, as recovery occurred. Because of the marked differences between disease characteristics in term compared to preterm infants, we studied these groups separately and compared both groups to a control group of infants without respiratory disease.

\section{METHODS \\ Collection of BAL samples}

Bronchoalveolar lavage (BAL) samples were collected from term (born $\geqslant 37$ weeks) and preterm (born <37 weeks) infants ventilated for RSV bronchiolitis. RSV status was ascertained by enzyme linked fluorescence (ELFA, Biomeriux) of nasopharyngeal aspirate. The control group comprised infants ventilated for non-infective and non-respiratory causes. BAL was performed according to recent ERS guidelines. ${ }^{18}$ Briefly, a suction catheter was passed down the endotracheal tube. Two $1 \mathrm{ml} / \mathrm{kg}$ aliquots of sterile normal saline were instilled separately down the suction catheter. Lavage fluid was recovered with constant suction pressure into a mucus trap. In RSV infected infants, BAL was performed during the first four days following intubation, or until the child was extubated. In control infants, BAL was performed once on each infant in the first 24 hours after intubation. This procedure was performed prior to routine bronchial toilet by one operator and was well tolerated. BAL aliquots were pooled, kept "on ice" and analysed within one hour. All samples were "vortexed" vigorously, then filtered through nylon monofilament gauze (Precision Textiles Ltd, Bury, UK).

Clinical characteristics were collected for each infant. They included the Paediatric Index of Mortality (PIM) score which provides information about likelihood of death for children on intensive care. ${ }^{19}$ the local paediatric research ethical committee approved the study and informed consent was obtained from the parents.

Abbreviations: $B A L$, bronchoalveolar lavage; $C L D$, chronic lung disease of prematurity; IQR, interquartile range; RSV, respiratory syncytial virus 
Table 1 Clinical characteristics

\begin{tabular}{llll}
\hline & Term & Preterm & Control \\
\hline Number of patients/males & $24 / 7$ & $23 / 12$ & $10 / 2$ \\
Mean (SD) admission weight, kg & $4.2(1.2)$ & $3.5(1.3)$ & $3.3(0.8)$ \\
Mean (SD) gestation at birth, weeks & $38.7(1.6)$ & $30.1(3.6)^{*}$ & $38.4(1.4)$ \\
Mean (SD) age on admission, weeks & $6.1(4.9)$ & $16.0(14.7)^{*}$ & $1.0(2.5)$ \\
Mean (SD) corrected age, weeks & $4.8(5.5)$ & $5.6(13.1)$ & $0.4(3.2)$ \\
Mean (SD) time from disease onset to intubation, days & $5.7(2.9)$ & $5.4(2.2)$ & - \\
Median (IQR) time ventilated, hours & $96(32)$ & $151(130) \dagger$ & - \\
Mean (SD) PIM score & $0.124(0.07)$ & $0.147(0.08)$ & - \\
\hline${ }^{*} p<0.01$ compared with term and control infants. & & & \\
$t p<0.01$ compared with term infants. & & & \\
\hline
\end{tabular}

\section{Total cell count calculation}

Standard techniques were used to assess cell viability and cell concentration. Briefly, a $10 \mu \mathrm{l}$ sample of BAL was mixed and stained with $10 \mu \mathrm{l}$ Trypan blue, which allowed sample cell viability and total cell concentration to be assessed using a Neubauer haemocytometer.

\section{Differential cell count}

A $10 \mu \mathrm{l}$ aliquot of BAL was smeared onto slides, air dried, fixed, and stained using Diff-Quick stain (Gamidore Technical Services Ltd, Abingdon, UK). Differential cell counts were obtained by counting 300 leucocytes using a standard light microscope under an oil immersion lens. Unidentifiable and necrotic cells were not counted.

\section{Statistics}

Where data were normally distributed, they were expressed as mean (SD); differences between groups were examined with Student's $t$ test. Where data were not normally distributed they were expressed as medians and interquartile ranges (IQR); differences between groups were examined using the Mann-Whitney rank sum test. Graphical data were presented in the form of box and whisker plots. All statistical tests were two tailed and a p value equal to or less than 0.05 was reported as statistically significant. Statistical calculations were performed using SPSS 10.0.5 statistical package (SPSS Inc., Chicago, IL).

\section{RESULTS}

During the 2000/01 and 2001/02 RSV seasons, 120 BAL samples were collected from 47 infants (term, 24; preterm, $23)$, ventilated for RSV bronchiolitis in the seven days following intubation (table 1). Fourteen of the 23 preterm infants had required ventilation in the neonatal period. Five preterm infants had chronic lung disease of prematurity (CLD) and two of these subsequently died from complications of bronchiolitis. Ten BAL samples were collected from 10 infants (all born at term) in the control group. BAL was performed on five infants before cardiac surgery and five infants before abdominal surgery. None of the infants in the study had received passive immunoprophylaxis for RSV bronchiolitis.
Table 1 presents the characteristics of the patients. Term and preterm infants with bronchiolitis differed in mean gestational age at birth (term, 38.7 (SD 1.6) weeks; preterm, 30.1 (SD 3.6) weeks) and median duration of ventilation (term, 96 (IQR 32) hours; preterm, 151 (130) hours). The median duration of ventilation in preterm infants was significantly longer even if the five infants with CLD were excluded (term, 96 (IQR 32) hours; preterm, 136 (125) hours, $p=0.03$ ). Preterm infants were older on admission to PICU than term infants (preterm, mean 16.0 (SD) 14.7 weeks; term, 6.2 (4.9) weeks), although when their ages were corrected for gestational age, there was no significant difference between the term and preterm groups (preterm, mean 5.6 (SD 13.1) weeks; term, 4.8 (5.5) weeks). There were no other differences between the term and preterm groups or the control group for any other features examined (table 1).

Table 2 presents the characteristics of the BAL samples. Values are expressed as median (IQR). The median total cell concentration over the seven days following intubation (fig 1) was significantly greater in term $\left(2.2(4.27) \times 10^{6}\right.$ cells $\left./ \mathrm{ml}\right)$ than preterm $\left(0.58(1.28) \times 10^{6}\right.$ cells $\left./ \mathrm{ml}\right)$ infants with RSV bronchiolitis or controls $\left(0.62(0.77) \times 10^{6}\right.$ cells $\left.\left./ \mathrm{ml}\right)\right)$. Neutrophil concentration was greater in term $\left(1.78(3.33) \times 10^{6}\right.$ cells/ $\mathrm{ml})$ than preterm $\left(0.42(1.22) \times 10^{6}\right.$ cells $\left./ \mathrm{ml}\right)$ infants with bronchiolitis or controls $\left(0.37(0.61) \times 10^{6}\right.$ cells $\left./ \mathrm{ml}\right)$. Alveolar macrophages were greater in term $\left(0.19(0.48) \times 10^{6}\right.$ cells $\left./ \mathrm{ml}\right)$ than preterm $\left(0.06(0.12) \times 10^{6}\right.$ cells $\left./ \mathrm{ml}\right)$ infants with bronchiolitis or controls $\left(0.09(0.10) \times 10^{6}\right.$ cells $\left./ \mathrm{ml}\right)$. Lymphocyte concentration was not significantly different between term $\left(0.08(0.22) \times 10^{6}\right.$ cells $\left./ \mathrm{ml}\right)$, preterm $\left(0.05(0.08) \times 10^{6}\right.$ cells $/ \mathrm{ml})$, and control $\left(0.05(0.12) \times 10^{6} \mathrm{cells} / \mathrm{ml}\right)$ groups (fig 1). There was no correlation between total or specific leucocyte cellularity and age or weight on admission. The analyses were repeated after five infants with a history of CLD were excluded from the preterm group; similar differences were observed (data not shown). Preterm infants with a history of CLD were also compared to those born preterm but without CLD. While those infants with a history of CLD were ventilated for significantly longer (preterm and CLD, 321 (324) hours; preterm, 136 (125) hours: $p=0.03$ ), there was no difference in the cellular responses over the first four days following intubation (data not shown).

Table 2 BAL characteristics

\begin{tabular}{llll}
\hline & Term & Preterm & Control \\
\hline Number of samples & 47 & 73 & 10 \\
Mean (SD) BAL recovery volume as a percentage of volume instilled & $41.9(22.9)$ & $54.2(20.5)^{*}$ & $47.0(22.3)$ \\
Mean (SD) cell viability, \% live cells & $77.8(17.2)$ & $80.0(17.0)$ & $68.8(18.7)$ \\
Median (IQR) percentage neutrophils & $85.3(12.7) \dagger$ & $78.0(16.0)$ & $52.3(27.7)$ \\
Median (IQR) percentage alveolar macrophages & $8.0(6.3) \dagger$ & $12.0(17.0)$ & $32.7(23.3)$ \\
Median (IQR) percentage lymphocytes & $6.0(4.3) \dagger$ & $8.0(7.0)$ & $11.0(13.0)$
\end{tabular}

${ }^{*} p<0.05$ compared with term and control infants.

$\mathrm{t} \mathrm{p}<0.05$ between all three groups. 


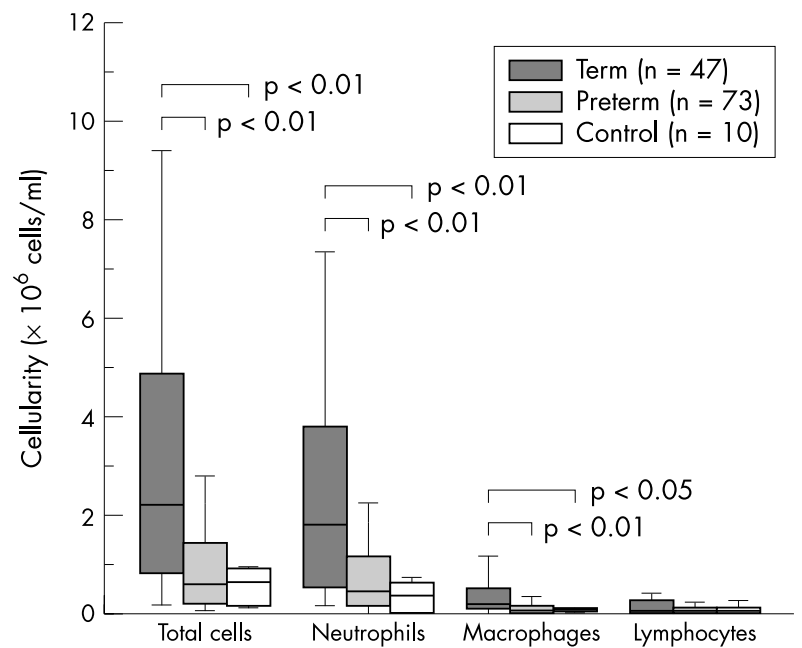

Figure 1 Total and individual leucocyte concentrations in term and preterm infants with bronchiolitis and the control group over days $1-4$. Eosinophils were only seen in six samples, where they constituted less than $1 \%$ of the total. Necrotic and unidentifiable cells were not counted. All respiratory epithelial cells seen were non-viable.

Table 2 shows individual cell type concentrations expressed as a percentage of the total cell count. The percentage of neutrophils in BAL was significantly greater in term (85.3\% (IQR $12.7 \%)$ ) compared to preterm $(78.0 \%(16.0 \%))$ infants with bronchiolitis, and both were greater than the control group $(52.3 \%(27.7 \%))$. Eosinophils were observed in only six samples from infants ventilated with bronchiolitis, where they comprised less than $1 \%$ of the total.

When the relation between BAL cellularity and time was examined in infants with bronchiolitis, total cell concentration decreased over the four days following intubation in term infants (fig 2). Total cell concentration was significantly greater on day $1\left(2.60(\right.$ IQR 8.62$) \times 10^{6}$ cells $\left./ \mathrm{ml}\right)$ than day 4 $\left(0.72(2.66) \times 10^{6}\right.$ cells $\left./ \mathrm{ml}\right)$ following intubation in term infants. Total cell concentration in preterm infants was similar throughout the period of ventilation, even up to day 7 (fig 2 ). Total cell concentration differed between term and preterm infants with bronchiolitis over the first three days following intubation. Variations in neutrophil concentration in infants with bronchiolitis over this period were similar to those observed for total cell concentration (fig 3).

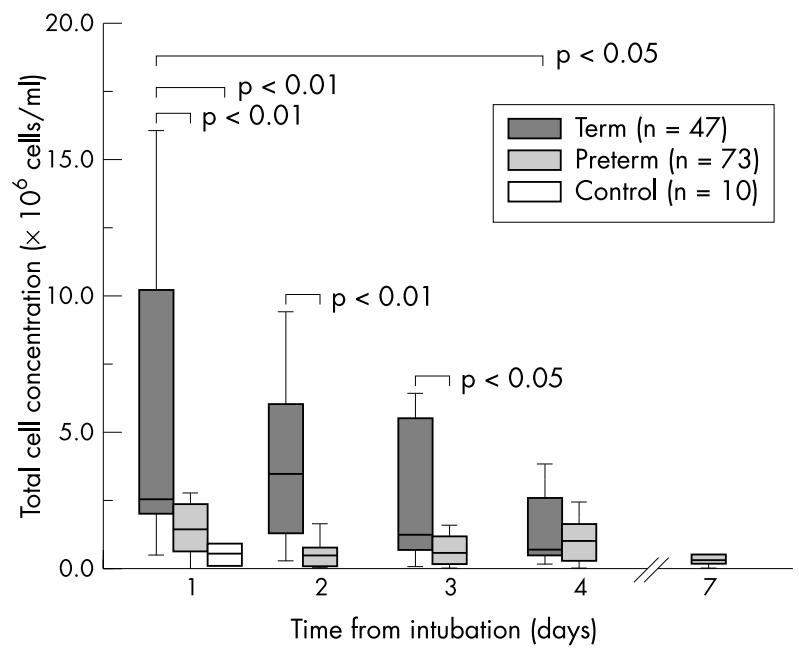

Figure 2 Total cell concentration over the first four days after intubation in term and preterm infants with RSV bronchiolitis. Data are also shown for control group infants (day 1) and six preterm infants still ventilated on day 7 .

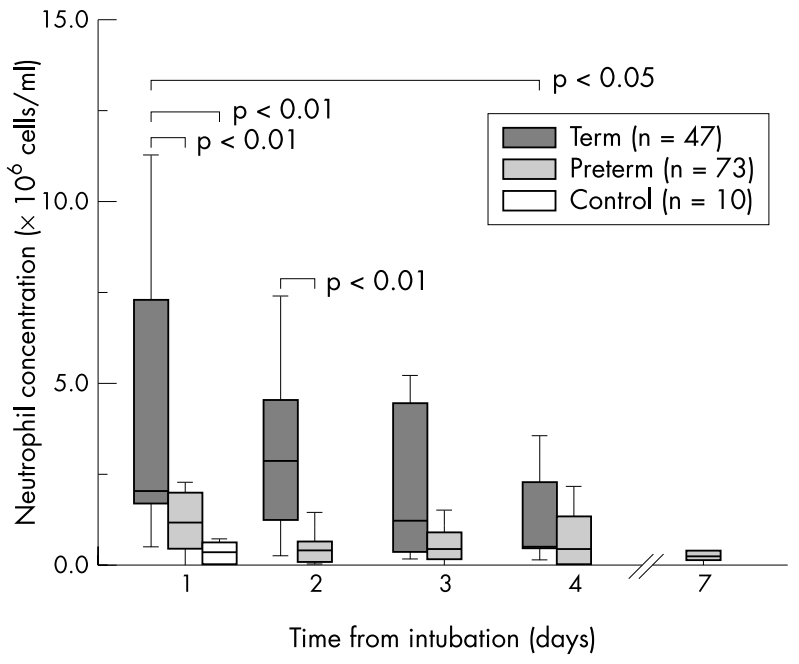

Figure 3 Neutrophil concentration over the first four days after intubation in term and preterm infants with RSV bronchiolitis. Data are also shown for control group infants (day 1) and six preterm infants still ventilated on day 7 .

\section{DISCUSSION}

This is the first study of which we are aware, to systematically quantify the pulmonary cellular response in term and preterm infants with RSV bronchiolitis over time. We have shown that the total cell concentration was greater in term compared to preterm infants with bronchiolitis and decreased in term infants from the time of intubation. We have also shown that variations in neutrophil concentration accounted for the majority of these changes. The magnitude of the cellular response in preterm infants with bronchiolitis was similar to that in the control group of infants without respiratory disease. Consistent with other studies, ${ }^{20}$ preterm infants with RSV bronchiolitis were ventilated for longer than term infants. Exclusion from the preterm group of those infants with a history of CLD did not affect these findings.

It was only possible to perform BAL on ventilated infants. Therefore, although we were particularly interested in the cellular profiles of infants with severe disease, we were unable to compare these to infants with milder disease. Despite this limitation, our study had a number of strengths. Firstly, BAL samples were collected in a systematic way, which minimised the effect of variable sample dilution inherent in other forms of sample collection from the lower respiratory tract. Secondly, we were able to compare these findings to a suitable control group of ventilated infants, who were similar in age, gestational age, and weight to the infants ventilated for RSV bronchiolitis. Thirdly, we included infants born preterm in our study. This group of children are often excluded from clinical studies, although a high proportion of preterm infants with RSV bronchiolitis develop critical airways obstruction, resulting in respiratory failure and ventilation. ${ }^{3}$

Recently, a new paramyxovirus associated with respiratory illness has been described. ${ }^{21}$ Human metapneumovirus (hMPV) causes clinical symptoms that are similar to illnesses caused by RSV, ranging from mild upper respiratory tract problems to severe cough, bronchiolitis, and pneumonia. ${ }^{21}$ Infection typically occurs during the winter months and is common during childhood, with most children having serological evidence of infection by 5 years. We have recently shown that a high proportion of infants with severe RSV bronchiolitis are co-infected with hMPV. ${ }^{22}$ The impact of this dual infection is not understood at the moment.

Few studies have examined the cellular response in infants with RSV bronchiolitis. Everard and colleagues ${ }^{14}$ found that the cellular infiltrate in nasopharyngeal secretions and BAL samples from infants with RSV bronchiolitis, comprised 
mainly neutrophils. More recently, Smith and colleagues ${ }^{15}$ showed that the total cell count from upper airway secretions was markedly higher than that obtained from peripheral blood in infants with bronchiolitis. Their study also showed that neutrophils are the predominant cell type in both RSV positive and RSV negative bronchiolitis. A recent randomised double blind, placebo controlled trial of dexamethasone treatment monitored pulmonary cellularity over time in 41 infants ventilated with severe RSV bronchiolitis. ${ }^{20}$ Total cell concentration in tracheal aspirates decreased significantly from the time of intubation. Treatment with dexamethasone made no difference to total cell concentration over this period in either the treatment or placebo groups. The same study also noted that preterm infants were ventilated for longer than those infants born at term.

Most information regarding the pathological changes in the lungs of infants born preterm comes from postmortem studies in infants with established CLD. The most significant changes are seen in the terminal bronchioles and alveolar ducts. ${ }^{23}$ Peribronchial fibrosis and obliterative fibroproliferative bronchiolitis occur in response to chronic airway inflammation. Smooth muscle hypertrophy and associated bronchospasm also occur and can cause small airways obstruction. Infants born preterm, who do not develop CLD, may have similar but less marked pathological changes in the lung. These changes may not become apparent until the child becomes infected with RSV.

In our study, the differences between term and preterm infants with RSV bronchiolitis in total cell and neutrophil concentration in BAL were particularly striking. There are a number of possible explanations for this. Differences in BAL cellularity may reflect gross structural abnormalities in the preterm lung. In preterm infants, the airways may be narrower and less compliant, and the distribution of instilled BAL fluid may therefore differ from term infants. The volume of BAL fluid recovered as a percentage of that instilled was significantly greater in preterm infants than either term infants with bronchiolitis or control infants without respiratory disease (table 2 ). It is possible that in preterm infants, instilled BAL fluid was held more centrally in the larger proximal airways and when suction was applied, relatively more fluid was recovered. If this were the case, less of the fluid instilled would reach the bronchioles, the site of the primary pathology, and fewer cells would be recovered.

Abnormal cellular recruitment from the bloodstream into infected airway may also explain the differences in BAL cellularity between term and preterm infants. Neutrophil recruitment into RSV infected lung tissue is a dynamic process. ${ }^{24}$ In RSV bronchiolitis, neutrophils migrate towards virus infected respiratory epithelial cells and alveolar macrophages along chemokine gradients. ${ }^{25}$ In preterm infants with RSV bronchiolitis, underlying pathological changes in the lung secondary to prematurity could impede the migration of cells from the pulmonary vasculature into the airway lumen.

Another reason for the difference in BAL cellularity relates to the amount of inflammation required to cause obstruction. In infants with bronchiolitis, the small airways are blocked by inflammatory cells, sloughed epithelium, and excessive mucus secretion. ${ }^{3}$ Because the airways of preterm infants are already narrowed, ${ }^{23}$ less inflammatory influx may be required to produce critical airway obstruction than in term infants. In essence, term infants have greater physiological reserve during an episode of RSV bronchiolitis. This concept would help explain the much higher proportion of preterm infants compared with term infants who require ventilation during an episode of RSV bronchiolitis. It also addresses the interplay between the immunological events in the lungs and their physiological consequences.

In term infants ventilated with bronchiolitis, the decrease in BAL cellularity following intubation may also be due to a number of factors. Cell concentration within the RSV infected airway is likely to peak around the time of intubation, when the infant is unable to clear his own obstructed airways. Once intubated, cellularity will decrease as cellular and mucus secretions are regularly suctioned from the lower respiratory tract and endotracheal tube. In addition, as the infant recovers, the cellular infiltrate within the airway is likely to diminish.

Neutrophils amplify the cellular response to RSV infection by secreting more chemokines, ${ }^{25}$ and recently the value of this response has been questioned. Neutrophils induce damage to respiratory epithelial cells infected with $\mathrm{RSV}^{26}$ and Hussell and colleagues $^{27}$ have suggested that they may also cause "bystander" damage to uninfected areas of lung. In term infants ventilated with RSV bronchiolitis, the neutrophil concentration decreases with time as the child's clinical condition improves. In preterm infants, where the cellular response is relatively constant throughout the duration of ventilation, the clinical course of the disease probably relates more to the severity of underlying lung disease than the vigour of the neutrophil response.

In conclusion, there are differences in the magnitude and type of pulmonary cellular response in term and preterm infants ventilated with RSV bronchiolitis. The cellular response in term infants with bronchiolitis also differs from that in a control group of infants. These differences may reflect variations in cellular recruitment into the airway and/or variations in airway calibre. Further work will investigate whether there are differences in the immunological responses between these groups of infants.

\section{ACKNOWLEDGEMENTS}

This work was supported by a grant from Action Research (grant ref. SP3814).

......................

Authors' affiliations

P S McNamara, R L Smyth, Department of Child Health, University of Liverpool, Liverpool, UK

C A Hart, Department of Medical Microbiology, University of Liverpool P Ritson, Physiotherapy Department, Alder Hey Children's Hospital, Eaton Rd, Liverpool

A Selby, Paediatric Intensive Care Unit, Alder Hey Children's Hospital

\section{REFERENCES}

1 Eriksson M, Bennet R, Rotzen-Ostlund M, et al. Population-based rates of severe respiratory syncytial virus infection in children with and without risk factors, and outcome in a tertiary care setting. Acta Paediatr 2002;91:593-8.

2 Martin AJ, Gardner PS, McQuillin J. Epidemiology of respiratory viral infection among paediatric inpatients over a six-year period in north-east England. Lancet 1978;2:1035-8.

3 Hall CB. Respiratory syncytial virus. In: Feigin RD, Cherry JD, eds. Textbook of paediatric infectious diseases. Philadelphia: WB Saunders, 1998:2084-111.

4 Openshaw PJ. Potential therapeutic implications of new insights into respiratory syncytial virus disease. Respir Res 2002;3:S15-20.

5 Navas L, Wang E, Robinson J. Improved outcome of respiratory syncytial virus infection in a high-risk hospitalized population of Canadian children. Pediatric Investigators Collaborative Network on Infections in Canada. J Pediatr 1992;1 21:348-54

6 Gavin R, Anderson B, Percival T. Management of severe bronchiolitis: indications for ventilator support. N Z Med J 1996;109:137-9.

7 de Sierra TM, Kumar ML, Wasser TE, et al. Respiratory syncytial virus-specific immunoglobulins in preterm infants. J Pediatr 1993; 122:787-91.

8 Nievas FF, .Chernick V. Bronchopulmonary dysplasia (chronic lung disease of infancy): an update for the pediatrician. Clin Pediatr (Phila) 2002;41:77-85.

9 Jobe AJ. The new BPD: an arrest of lung development. Pediatr Res 1999;46:641-3.

10 Kimpen JL, Ogra PL. T cell redistribution kinetics after secondary infection of BALB/c mice with respiratory syncytial virus. Clin Exp Immunol 1993;91:78-82.

11 Openshaw PJ. Flow cytometric analysis of pulmonary lymphocytes from mice infected with respiratory syncytial virus. Clin Exp Immunol 1989;75:324-8.

12 Taylor G, Stott EJ, Hayle AJ. Cytotoxic lymphocytes in the lungs of mice infected with respiratory syncytial virus. J Gen Virol 1985;66 (pt 12):2533-8. 
13 Taylor G, Thomas LH, Stott EJ. Effect of vaccination on cell populations in lung washes from calves after infection with respiratory syncytial virus. Res Vet Sci 1989;47:231-5.

14 Everard ML, Swarbrick A, Wrightham M, et al. Analysis of cells obtained by bronchial lavage of infants with respiratory syncytial virus infection. Arch Dis Child 1994;71:428-32.

15 Smith PK, Wang SZ, Dowling KD, et al. Leucocyte populations in respiratory syncytial virus-induced bronchiolitis. J Paediatr Child Health 2001;37:146-51.

16 Aherne W, Bird T, Court SD, et al. Pathological changes in virus infections of the lower respiratory tract in children. J Clin Pathol 1970;23:7-18.

17 Kim HW, Canchola JG, Brandt CD, et al. Respiratory syncytial virus disease in infants despite prior administration of antigenic inactivated vaccine. Am J Epidemiol 1969;89:422-34.

18 de Blic J, Midulla F, Barbato A, et al. Bronchoalveolar lavage in children. ERS Task Force on bronchoalveolar lavage in children. European Respiratory Society. Eur Respir J 2000;15:217-31.

19 Shann F, Pearson G, Slater A, et al. Paediatric index of mortality (PIM): a mortality prediction model for children in intensive care. Intensive Care Med 1997;23:201-7.
20 Buckingham SC, Jafri HS, Bush A et al. A randomized, double-blind, placebo-controlled trial of dexamethasone in severe respiratory syncytial virus (RSV) infection: effects on RSV quantity and clinical outcome. J Infect Dis 2002;185:1222-8

21 van den Hoogen BG, de Jong JC, Groen J, et al. A newly discovered human pneumovirus isolated from young children with respiratory tract disease. Nat Med 2001;7:719-24.

22 Greensill J, McNamara PS, Dove W, et al. Human metapneumovirus in severe respiratory syncytial virus bronchiolitis. Emerg Infect Dis 2003;9:372-5

23 Cherukupalli K, Larson JE, Rotschild A, et al. Biochemical, clinical, and morphologic studies on lungs of infants with bronchopulmonary dysplasia. Pediatr Pulmonol 1996;22:215-29.

24 Wang SZ, Hallsworth PG, Dowling KD, et al. Adhesion molecule expression on epithelial cells infected with respiratory syncytial virus. Eur Respir J 2000;15:358-66.

25 Wang SZ, Forsyth KD. The interaction of neutrophils with respiratory epithelial cells in viral infection. Respirology 2000;5:1-10.

26 Wang SZ, Xu H, Wraith A, et al. Neutrophils induce damage to respiratory epithelial cells infected with respiratory syncytial virus. Eur Respir J 1998;12:612-18.

27 Hussell T, Pennycook A, Openshaw PJ. Inhibition of tumor necrosis factor reduces the severity of virus-specific lung immunopathology. Eur $J$ Immunol 2001;31:2566-73.

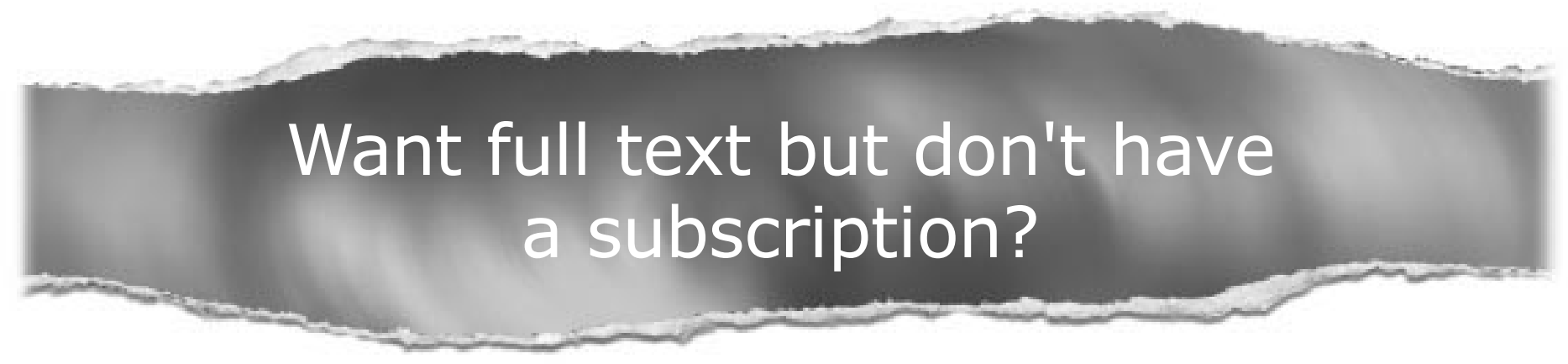

\section{Pay per view}

For just \$8 you can purchase the full text of individual articles using our secure online ordering service. You will have access to the full text of the relevant article for 48 hours during which time you may

download and print the pdf file for personal use.

www.archdischild.com 